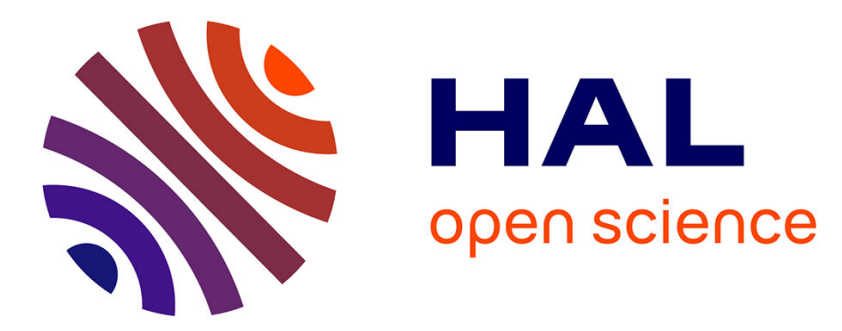

\title{
Vécu au travail des internes de médecine: étude qualitative
}

\author{
R. Pougnet, L. Pougnet, J.-D. Dewitte, D. Jousset, B. Loddé
}

\section{To cite this version:}

R. Pougnet, L. Pougnet, J.-D. Dewitte, D. Jousset, B. Loddé. Vécu au travail des internes de médecine: étude qualitative. Archives des Maladies Professionnelles et de L'Environnement, 2019, 80, pp.100 107. 10.1016/j.admp.2018.11.001 . hal-03486360

\section{HAL Id: hal-03486360 https://hal.science/hal-03486360}

Submitted on 20 Dec 2021

HAL is a multi-disciplinary open access archive for the deposit and dissemination of scientific research documents, whether they are published or not. The documents may come from teaching and research institutions in France or abroad, or from public or private research centers.
L'archive ouverte pluridisciplinaire HAL, est destinée au dépôt et à la diffusion de documents scientifiques de niveau recherche, publiés ou non, émanant des établissements d'enseignement et de recherche français ou étrangers, des laboratoires publics ou privés.

\section{다)(1) $(5$}

Distributed under a Creative Commons Attribution - NonCommerciall 4.0 International 
Version of Record: https://www.sciencedirect.com/science/article/pii/S1775878518308476

Manuscript_bd25735611cc41a794cb3b1cef28c13f

\section{Article ADMP 2018_90}

Vécu au travail des internes de médecine : étude qualitative

Feeling at the work of junior doctors: qualitative study

Richard Pougnet ${ }^{1,2}$, Laurence Pougnet ${ }^{3}$, Jean-Dominique Dewitte ${ }^{1,2}$, David Jousset ${ }^{4}$, Brice Loddé $^{5,6}$

Conflit d'intérêt : aucun

(C) 2018 published by Elsevier. This manuscript is made available under the CC BY NC user license https://creativecommons.org/licenses/by-nc/4.0/ 
Vécu au travail des internes

Résumé :

Introduction: les internes en médecine constituent une population à risque de troubles réactionnels au travail. L'objet de cette étude était de qualifier par la narration les facteurs perçus comme positifs et ceux perçus comme négatifs au travail pour les internes d'une université française.

Matériel et Méthode: il s'agissait d'une étude qualitative, selon la théorie ancrée par thématisation continue, auprès d'internes de médecine (générale et spécialisée).

Résultats: La variation maximale, en fonction de critères professionnels et sociaux, et la saturation des données de codage ont été atteintes après 10 entretiens non-dirigés. Les internes ont exprimé l'importance des relations au travail, au dessus de toute autre dimension professionnelle, qu'il s'agisse des relations avec les autres médecins (tuteurs ou autres internes) que celles avec les paramédicaux. Les difficultés organisationnelles et la charge de travail semblaient passer au second plan, et surtout se dérouler lors des stages en hôpital universitaire.

Conclusion: Ces données permettaient de mieux considérer les résultats des études quantitatives et de mieux comprendre le vécu des internes en médecine. Des axes de prévention peuvent se dégager pour les universités, les hôpitaux et les médecins accueillants des internes.

Mots-clés : Internes en médecin ; Etude Qualitative ; Relations professionnelles ; vécu au travail. 
Vécu au travail des internes

Abstract :

Introduction: Junior doctors constitute a population at risk of reactive disorders at work. The purpose of this study was to describe the factors perceived as positive and those as negative at work for the interns of a French university.

Material and Method: this was a qualitative study, according to the Grounded theory, among medical interns (general and specialized).

Results: Maximum variation, based on occupational and social criteria, and saturation of coding data were achieved after 10 non-directed interviews. The junior doctors expressed the importance of relationships with other professionals (ie, physicians or other junior doctros) than those with paramedics. Organizational difficulties and workload seemed to take a back seat, but these factors especially took place during university hospital internships.

Conclusion: These data made it possible to better consider the results of quantitative studies and to better understand the experience of residents in medicine. Prevention axes can be identified for universities, hospitals and physicians.

Keywords: "Physicians"[Mesh]; "Qualitative Research"[Mesh]; "Interprofessional Relations"[Mesh]; "Quality of Life"[Mesh] 
Vécu au travail des internes

Introduction :

Les internes en médecine exercent, en France, dans les hôpitaux et autres lieux de stage, entre la $7^{\mathrm{e}}$ et la dernière année de leurs études de médecine ; la durée de ces études étant variable selon le type de spécialité [1]. Pendant leurs internats, ils sont à la fois médecins, sous la responsabilité d'un médecin «sénior», ayant eu l'ensemble de ses diplômes et étant en exercice, et à la fois étudiants. Ils doivent ainsi conjuguer le travail de médecin et l'approfondissement de leurs connaissances et la poursuite d'études scientifiques pour rédiger leur thèse de médecine et leur mémoire de spécialité.

Cette charge de travail, ces injonctions parfois paradoxales entre l'hôpital et la faculté, cette mise en situation sans avoir toujours le savoir-faire requis, peuvent engendrer des situations conflictuelles, ou bien susciter des interrogations sur le travail, son sens, et sa capacité à l'affronter. La prévalence des troubles réactionnels, notamment anxieux et de l'humeur, est très élevée dans cette population. La prévalence des troubles anxieux pouvait s'élever à 56,3\% dans certaines études portant sur l'ensemble des internes; celle du trouble de l'humeur réactionnelle, à $48 \%$; et celle du syndrome d'épuisement professionnelle (burn out, $\mathrm{BO}$ ), $74 \%$, voire $80,2 \%$. Ces estimations variaient d'un pays à l'autre et d'une spécialité médicale à l'autre [2-5]. En France, les prévalences des troubles anxieux, de l'humeur et du BO ont été estimées à $28,7 \%, 13 \%$ et $44 \%$, contre, en population générale, 17 à $25 \%$ de troubles anxieux et 7,5\% de trouble de l'humeur chez les 18-85 ans. [5-7].

Les approches souvent quantitatives de ces problématiques de santé ne permettaient pas toujours de dégager les facteurs ressentis comme les plus difficiles, ni les représentations des internes quant à leur rôle. Une réforme de l'internat est en cours en France qui changera le métier d'interne, avec notamment une intégration progressive dans le soin. Actuellement, 
Vécu au travail des internes

selon les stages, les internes peuvent en effet se voir confier la responsabilité d'un service quasiment sans contrôle des médecins séniors. L’objet de cet article est d'étudier la manière dont les internes, qui ne bénéficient pas encore de la réforme française, perçoivent leur travail, tant en ce qui leur paraît être positif que négatif.

Matériel et Méthode :

Il s'agissait d'une étude qualitative par entretiens non dirigés. Les critères d'inclusion étaient d'être interne de médecine générale ou d'une spécialité médicale dans la subdivision de Brest, Bretagne Occidentale. Etaient exclus les internes en chirurgie. L'étude s'est déroulée entre fin 2016 et début 2017, avant la mise en place des réformes de l'internat français.

Les participants étaient sélectionnés selon une méthode d'échantillonnage raisonnée, pour atteindre la variation maximale, par la technique de proches en proches et par tirage au sort à partir de la liste des internes donnée par la scolarité. Les étudiants tirés au sort pouvaient donc orienter l'enquêteur directement vers un de ses co-internes intéressé par l'étude. Les internes recevaient une information sur l'étude, sa modalité, ses finalités, et sur la confidentialité. Ils donnaient leur accord ou non pour participer à cette étude, après contact téléphonique ou direct. La taille de l'échantillon n'était pas définie préalablement. Le critère était d'atteindre la variation maximale selon les données sociodémographiques et professionnelles, puis la saturation des données [8,9]. La variation maximale se définissait comme étant le fait d'avoir interrogé au moins un interne de chaque sexe, de chaque année, de chaque grand type de disciplines médicales (médecine générale, médecine spécialisée), ayant fait un stage en différentes institutions (CHRU, autre hôpital, clinique, cabinet libéral), ayant changé de spécialité pendant son cursus, ayant redoublé ou non, ayant eu des enfants ou non. La 
Vécu au travail des internes

saturation des données correspondait au fait qu'aucun nouveau thème n'apparaissait sur 2 entretiens de suite.

Les interviews se faisaient en face à face dans le bureau de l'enquêteur, ou bien dans le lieu choisi par le participant, à condition que ce lieu fût calme et assurât que les interviews ne soient pas interrompues. Les entretiens se faisaient de manière non dirigée. Seule une question modale large (« que trouves-tu de positif ou négatif dans ton travail d'interne ? ) était posée. L'enquêteur utilisait plusieurs techniques de relances et de reflet, ou de reformulation pour assurer le déroulement de l'entretien. Les entretiens étaient enregistrés sur un dictaphone (Olympus $^{\odot}$ ), puis retranscrit sur fichier $\operatorname{Word}^{\odot}$ par l'enquêteur; les logiciels d'autodictée ayant été écartés en raison du risque de mauvaise compréhension des paroles. L'anonymat des entretiens étaient assuré puisque le nom du participant n'était pas enregistré. L'enquêteur a, au besoin, effacé tous les noms cités lors des entretiens au moment de la retranscription sur tapuscrit.

Les entretiens étaient ensuite analysés selon la théorie ancrée par thématisation continue. En d'autres termes, le codage était progressif en trois temps. Le codage ouvert repérait systématiquement tous les éléments importants évoqués dans chaque verbatim. Puis, le codage axial consistait à regrouper en catégories les codes ouverts. Enfin, les thèmes principaux étaient dégagés par le codage sélectif, en analysant l'ensemble du corpus des verbatim et de leur analyse. 
Vécu au travail des internes

Résultats :

La variation maximale et la saturation des données ont été atteintes après 10 entretiens (tableau $\mathrm{n}^{\circ}$ ). Les entretiens ont duré entre 23 minutes et 1 h59. Les principaux thèmes étaient les relations avec les médecins, celles avec les équipes paramédicales et avec les autres internes (ou co-internes), puis la charge de travail en stage et les études (figure $\mathrm{n}^{\circ} 1$ ).

a) Ambivalences des relations avec les médecins séniors :

Il s'agissait du thème le plus récurrent dans les verbatim.

Souvent perçues comme difficiles, elles pouvaient se révéler très conflictuelles, certains relataient des vexations répétées : «j'ai été humilié par le chef de service... Dès le début, dès la première semaine »; «je me mettais toujours dans la position où j'étais l'élève et lui le maître. Même quand je n'étais pas d'accord, je faisais comme il disait. C'était lui l'autorité et moi j’avais rien à dire »; «on savait que les gardes avec lui...Personne ne voulait travailler avec lui (...) J'avais toujours la boule au ventre : il était très caractériel. » Un des internes avait développé des troubles anxieux réactionnels aux relations avec son chef de service ; il en avait résulté une dépendance à l'alcool, les consommations se faisant dans un but anxiolytiques le soir. Plus couramment, les propos étaient vécus comme déplaisants et peu constructifs : «il y avait toujours des remarques pour dire ce qui n'allait pas, mais jamais une remarque positive pour dire ce qui était bien ».

Pourtant, les internes étaient très ambivalents à propos de ces relations. Ils pouvaient y trouver de la réassurance («je ne me suis jamais sentie en détresse parce qu'il y a toujours un PH, au moins dans le secteur d'à côté. ») ou de l'aide («ils comprennent bien que l'interne n'a pas le droit de tout faire... donc ils n'envoient pas [sur les roses]»), tout en reconnaissant que la 
Vécu au travail des internes

formation ne se faisait pas sans douleur («je me rends compte, après coup, que j'ai appris plein de choses... Mais ça a été vraiment très difficile »). Ainsi, un même interne pouvait avoir vécu ses relations de manière positives sur le plan de l'aide et de la formation, mais négatives du point de vue affectif.

Les internes pensaient surtout que la disponibilité des médecins était le catalyseur d'un bon climat de travail. Ils pensaient que les stages en dehors des CHRU étaient plus formateurs et plus agréables, les médecins y étant plus à l'écoute de leurs questionnements et moins en demande de productivité de soins. Selon eux, les médecins des stages en petit hôpital ou en milieu libéral étaient habitués à ne pas travailler avec un interne et que, en conséquence, le travail de l'interne était perçu comme une aide appréciable. Ils soulignaient également le rapport hiérarchique plus marqué en CHRU («à l'hôpital, il y a une hiérarchie ») que dans d'autres lieux de stage («c'est plus horizontal. On est plus sur un pied d'égalité »). La disponibilité des médecins conditionnait ainsi le vécu du stage et corollairement le choix du lieu de stage par les internes: «c'est assez flagrant, t'as des stages où les PH sont assez dispo, te montrent des trucs»; «c'est clair et net que les choix de stages...sont...sont totalement fonction de l'implication des PH dans leurs services ».

Pourtant, les internes étaient ambivalents. Ils percevaient le maître de stage (médecin responsable) soit comme trop peu impliqué, les laissant assumer des responsabilités au-dessus de leurs compétences («j'ai été lâchée parce qu'il n’y avait pas de chef. Elle était malade. Du coup, j'l'ai remplacée toute seule»), soit comme une personne qui ne leur laissait pas suffisamment d'autonomie («je la trouvais trop maternante; j'avais envie de me lancer»; «je me sentais frustrée alors que je me sentais de le faire »). Ces ressentis pouvait se voir chez un même interne, parfois au décours d'un même stage. Les internes appréciaient particulièrement d'être impliqués par le sénior, à hauteur de leur ancienneté, dans le soin ; en d'autres termes, les médecins qui leur demandaient réellement leur avis, qui les 
Vécu au travail des internes

reconnaissaient comme de futurs confrères ayant déjà du savoir-faire, étaient perçus comme encourageants : "on n'impose pas, on est dans la relation et l'alliance ».

b) Lutte de pouvoir avec les paramédicaux :

Les internes exprimaient de nombreuses difficultés à s'intégrer dans les équipes de soins. Les relations avec les paramédicaux étaient souvent perçues comme une lutte de pouvoir, ou une remise en question de leurs compétences médicales («Les rapports de forces... On est là 6 mois, on débute, alors que elles, elles sont là depuis un moment »). Les internes devaient apprendre à se positionner en tant que médecins dans des collectifs de travail déjà constitués («j’ai eu du mal à discuter avec les équipes. J'avais du mal aux transmissions le matin, à affirmer que c'était moi le médecin»), et ayant leur propre histoire, passée et à venir sans l'interne en question. Certaines habitudes de service pouvaient paraître déviantes aux yeux de l'interne, avec des protocoles parfois éloignés des habitudes d'autres services. L'interne ne connaissant pas l'histoire des équipes et la genèse de ces consensus organisationnels pouvait alors se trouver en position de déviant, plus ou moins constant; des tensions interrelationnelles pouvaient alors réapparaître, ou bien se focaliser sur l'interne. «Les relations avec les équipes, ça m'a reboutée. On peut avoir un mauvais semestre juste à cause des tensions entre les chefs et l'équipe infirmière... Les problèmes de relations. Tout se dit par derrière ou par l'intermédiaire de l'interne ».

Certains internes trouvaient que «travailler avec l'équipe, c'est lourd, mais c'est très intéressant », dans la mesure où cette coopération passagère les remettaient eux-mêmes en question d'un point de vue professionnel (sur le plan de la technique médicale, ou des relations avec les patients et les équipes). 
Vécu au travail des internes

c) Les co-internes comme soutien hospitalier et estudiantins :

Les rapports avec les autres internes permettaient souvent de trouver des ajustements dans les relations avec les équipes médicales ou paramédicales. Les internes connaissant déjà le service servaient de référence, tant sur le plan technique et organisationnel, que sur le plan humain pour mieux se comprendre. Ils se soutenaient («C'est agréable de pouvoir parler») et exerçaient une fonction cathartique, en particulier lors des sorties et soirées en commun («ma bouffée d'air, c'est les tonus » [Note : un tonus est un fête réservée aux internes]).

Principalement, les internes pouvaient être des référents pour les plus jeunes : «il y avait deux filles de $4^{e}$ semestre, que je ne connaissais pas du tout. Elles ne faisaient pas du tout pareil, elles ne voyaient pas les choses de la même manière. C'était marrant. C'était intéressant de voir comment les autres bossent ».

d) Les stages hospitaliers, sources de satisfaction et de déplaisirs :

Le travail à l'hôpital s'associait à des champs lexicaux très ambivalents et contrastés. Bien que source d'épanouissement professionnel en tant que les internes y réalisaient ce pour quoi ils étaient formés, cet exercice s'accompagnait d'une grande anxiété au sujet de leur capacité à mettre en œuvre le savoir acquis tout au long des études. «Du jour au lendemain, tu passes d'externe à interne; tu dois être au point sur les prescriptions, les posologies et tout ça. Bon les équipes sont au courant que t'es en premier semestre ».

Selon la taille des structures et la disponibilité des médecins séniors, le travail leur paraissait comme plaisant ou déplaisant. Les stages en $\mathrm{CH}$ ou en cabinet étaient souvent perçus comme formateurs et reposants, tandis que les stages en CHRU étaient plus anxiogènes. Dans les hôpitaux périphériques, par exemple, "les médecins du service donnaient des cours d'une 
Vécu au travail des internes

heure, une heure et demie " et "il y avait une discussion avec les séniors, à propos de mes dossiers ». A l'inverse, au CHRU, les problèmes organisationnels y étaient plus fréquents : effectifs insuffisants («on était moins d'internes que prévu»); charges de travail trop importantes («le plus dur, c’était les horaires. Il arrivait qu'on fasse plusieurs week-ends de suite. Entre les gardes d'urgences et les astreintes de services... Quand t'enchaînes 21 jours de suite [au CHU], ça tire pas mal»; «on n'avait pas le respect des horaires... C'est combien 52 heures ? (...) On faisait plus. En plus, on avait les gardes et les astreintes »); ou encore matériel inadapté («dans un même bureau, on était 3, 4 internes...Oui, 6 externe et le chef (...) T'avais pas d'ordi. T'étais obligé d'attendre que ton collègue finisse »).

Toutefois le travail en CHRU pouvait s'avérer plus intéressant de part la complexité et la technicité des prises en charges: «c'est intéressant à l'hôpital, les pathos. Mais c'est compliqué », au point que certains internes de spécialité fassent des gardes au CHRU pendant des stages en périphérie ou pendant des stages de master de science : "j’ai demandé [à faire des gardes] parce ce que ça me manquait ». Certaines organisations de stage étaient très aidantes pour les internes enceintes, particulièrement si les chefs de services adaptaient les contraintes physiques ou les horaires selon les besoins, ou encore s'il était prévu d'affecter l'interne en effectif surnuméraire, dit surnombre : «j'avais prévu d'aller à [tel endroit] parce que je savais que j'étais enceinte et que je serai en surnombre ».

e) Trop peu de temps pour les études :

Le travail à l'hôpital semblait empêcher l'accès aux formations. Les internes devaient souvent rester dans les services au lieu d'aller assister aux cours. Souvent source de frustration pour l'interne, cela pouvait devenir un lieu de tensions avec les médecins : «régulièrement, on est 
Vécu au travail des internes

obligé de rappeler que l'interne a des cours et des formations ». Cela dépendait principalement de la taille de la structure : «alors que quand j'étais [dans un autre hôpital] on nous laissait y aller. On nous disait, même quand on manquait de médecin : ben non, vous êtes sensés y aller, alors vous y allez [aux cours de DES] »

L'attitude des médecins pouvait se révéler tout autre et véritablement aider les internes pour leurs formations: "j’étais tombé sur un PH sympa qui m'a donné des indications méthodologiques ». 
Vécu au travail des internes

Discussion :

Cette étude qualitative auprès des internes a permis de montrer les dimensions pénibles et stimulantes de leur vécu au travail. Alors que la question était ouverte, les propos ont principalement porté sur les relations au travail, particulièrement avec les médecins responsables de services. Le rôle des médecins dits séniors semblait prépondérant pour leur qualité de vie au travail et pour leurs études. De manière générale, les relations au sein du CHRU étaient plus délicates et pénibles. Il en était de même pour les relations avec les équipes paramédicales, les internes éprouvant des difficultés à trouver leur place dans un collectif de travail déjà institué, ou ressentant le regard des paramédicaux comme une remise en question de leurs compétences professionnelles.

L'approche qualitative semblait pertinente pour mieux comprendre le vécu au travail de cette population pour laquelle il existait déjà une littérature quantitative importante. La principale limite de cette étude résidait dans son approche des médecins de spécialités médicales et non chirurgicales. L'exclusion des internes en chirurgie était justifiée par la trop grande disparité en France des modalités d'exercice et d'études entre les spécialités médicales et chirurgicales. Une autre étude devrait porter sur cette population. Le fait d'avoir interrogé moins d'internes de médecine générale que d'internes de médecine d'autres spécialités, alors que les premiers sont les plus nombreux à l'internat, ne constituait pas une limite pour l'interprétation selon la théorie ancrée par thématisation continue. En effet, le critère de qualité d'une telle étude est la dispersion des sujets, à savoir le fait d'avoir au moins deux sujets de chaque catégorie, l'approche n'étant pas quantitative. La taille de l'échantillon était adaptée pour ce type de méthodologie. Toutefois, cette étude a été réalisée dans une seule subdivision de formation, à savoir une seule zone géographique, dépendant d'une seule faculté et attachée à un seul 
Vécu au travail des internes

CHRU. Les données ne sont pas nécessairement extrapolables à l'ensemble des internes français. En fonction des politiques pédagogiques ou des situations démographiques au sein des hôpitaux, d'autres thèmes auraient pu surgir lors d'entretiens avec des internes d'autres subdivisions. De plus, les données montraient une très grande variabilité du vécu des internes, d'un sujet à l'autre, mais aussi pour un même sujet selon les lieux de stages et l'évolution temporelle, ce qui rejoint la littérature [10]. Cette étude ne montrait donc l'état des internes qu'à un moment donné. Si un nouveau travail, particulièrement quantitatif, devait être réalisé, il conviendrait donc de prendre en compte la rapidité des changements chez les internes, d'un semestre à l'autre et au cours de leur cursus. De même, il existe peut-être un biais de recrutement : l'étude faisait appel aux internes. Il était donc possible que les internes les plus en souffrance et les internes les plus épanouis n'eussent pas la volonté ou la force d'y participer. Seule une étude quantitative permettrait d'avoir une évaluation sur la population source. La force de cette étude reposait sur l'expérience de l'enquêteur, à la fois médecin et formé en sciences humaines. Ainsi les concepts implicites de références, notamment les notions médicales et les modalités de gardes ou d'astreintes, les rôles joués par les acteurs, étaient partagés entre les personnes interrogées et celle qui menait les entretiens. L'analyse s'est déroulée en plusieurs temps de codage successif en conservant le corpus initial ; il n'a pas été fait de protothématisation (ou thématisation préalable et a priori), ce qui garantit une plus grande fidélité à ce qui avait été exprimé par les internes, à savoir un niveau d'inférence bas [9].

Certains thèmes ne sont pas ressortis au cours des entretiens, notamment la question de l'équilibre à trouver entre la vie privée et la vie professionnelle qui pose souvent de nombreuses difficultés aux internes et aux médecins [5,11]. Il est possible que cela tienne de l'environnement breton. Par exemple, le niveau de vie d'un interne en Bretagne Occidentale peut être supérieur à celui d'autres villes, l'activité peut y être à la fois urbaine ou rurale, ce 
Vécu au travail des internes

qui expliquerait que des thèmes comme les revenus ou les transports ne soient pas apparus dans cette étude. Ou encore, les internes ayant eu des enfants n'ont pas rapporté de difficultés à propos du mode de garde, alors que les horaires d'internes sont atypiques. Une étude est actuellement en cours pour mieux analyser cela. A contrario, certaines idées étaient singulières et pourraient favoriser un surinvestissement ou une minorisation du mal-être au travail chez les internes. Notamment, certains internes pensaient qu'il était normal de souffrir en apprenant : «la formation ne se fait pas sans douleur». Il serait utile confronter ce point de vue avec les éventuels bénéfices de la formation, comme la satisfaction d'apprendre de nouveaux gestes, de mettre en application ce que l'on a appris, ou encore la satisfaction relationnelle avec les patients. Une évaluation de l'équilibre effort-récompense pourrait être utile, d'autant que des actions ont pu être mises en place dans certains pays [12,13]. Mais plus généralement, la satisfaction au travail des internes peut être un axe de politique de santé. Au Royaume-Uni, les études ont montré que de plus en plus de médecins songeaient à arrêter la médecine ou à changer de pays [14]. Face à ces constats, plusieurs politiques de prévention se sont focalisées sur le bien-être au travail des médecins, et en particulier, des internes dont le programme de formation a changé en 2009 [15]. Il existe par exemple des programmes pour renforcer leur sentiment de compétence en fonction des lieux de stages; certains auteurs proposent de renforcer les activités de loisirs $[15,16]$.

Dans cette étude, la qualité des relations avec les autres internes soutenait les personnes lorsqu'elles rencontraient des difficultés pour s'intégrer ou des situations médicalement difficiles. Ces données s'opposaient aux résultats d'une étude quantitative menée sur des promotions précédentes, dans la même région. La compétition entre internes y était un facteur de risque de trouble anxieux : $\mathrm{OR}=4.23(1.06-16.82)$ [17]. En comparant les études, il apparaît que les relations de compétitions n'opposaient que les internes au sein d'une même spécialité pour l'obtention d'un même poste. Ceci avait également été trouvé dans d'autres 
Vécu au travail des internes

études, comme celle de Oschmann E et al $(\mathrm{p}<0,05)$ [18]. Golub et al ont montré également un lien entre le syndrome d'épuisement professionnel et la mauvaise qualité des relations entre internes : $\mathrm{r}=0,38 ; \mathrm{p}<0,001$ [19]. La réalité des relations entre internes se complexifierait donc selon les orientations et les trajectoires de carrière. Il en découle, selon nous, que cette relation, perçue généralement comme un soutien et une aide opérationnelle pour les études et les soins à l'hôpital, peut devenir une rivalité en fonction des organisations et des institutions. Cette étude qualitative permettait de souligner l'importance de cette relation aux yeux des internes eux-mêmes.

Dans certaines études quantitatives, le passage entre étudiant et interne est montré comme un épisode charnière à risque de BO. Une étude récente montrait une prévalence de $37 \%$ de BO suite à ce passage [20]. Bien que les internes de lère année n'aient pas été interrogés, l'analyse du verbatim de cette étude permettait de mieux comprendre les dynamiques de ce passage : la prise de responsabilité, le fait de ne pas avoir complètement terminé sa formation, le manque de clarté des interactions et supervisions de certains médecins séniors, et l'insertion dans l'équipe de soins étaient les principaux facteurs de vécu stressant au travail. L'insertion dans un collectif pluri professionnel et aux interactions multiples n'était pas aisé ; ceci rejoignait les données de la littérature quantitative et qualitative [21]. Dans certaines études ethnographiques, le manque de communication et la différence de représentation du soin entre les médecins et les infirmiers constituait le principal frein à la bonne insertion des internes [22]. Une piste de prévention serait alors une meilleure formation des internes pour l'insertion professionnelle. Certains auteurs ont proposé la mise en place de debriefing [23]. Le fait qu'une institution mette en place des espaces de communication pourrait être en effet utile.

Parallèlement, il serait logique de se demander si le classement de l'interne ne conditionnerait pas en partie sa bonne insertion en stage. En effet, selon le classement, l'interne a plus ou moins de choix entre les lieux de stage. Or, les internes de cette étude semblaient parfois 
Vécu au travail des internes

choisir leur stage en fonction de l'accueil fait aux internes. Les internes en fin de classement pourraient donc se voir affecter, de manière imposée, des lieux de stages moins propices à un bon déroulement de leur semestre. Cette étude ne permettait pas d'explorer l'effet du classement sur le ressenti du travail des internes, pour des raisons pratiques de recrutement des sujets. Il serait utile de faire un travail d'analyse sur ce facteur pour en dégager éventuellement des axes de prévention.

Le nouvel internat intégrera progressivement les étudiants dans le milieu du soin. En première année, ils devront apprendre les connaissances «socles » de leurs spécialités, et n’exerceront la médecine que sous le regard vigilant des médecins séniors. Ensuite, entre la $2^{\mathrm{e}}$ et l'antépénultième année, ils apprendront de plus en plus les gestes techniques en lien avec les médecins. Ce ne sera qu'en dernière année, après l'obtention de la thèse et avant la soutenance de mémoire de spécialité, qu'ils exerceront en autonomie ; mode d'exercice plus ou moins comparable avec celui des internes actuels. Un certain nombre de situations difficiles devraient donc disparaître. Les internes, par exemple, ne pourront plus, a priori, être mis en situations d'autonomie avant d'avoir eu le temps d'acquérir les connaissances spécifiques et le savoir-faire propres aux spécialités.

Mais d'autres situations devraient toujours se voir. Le délicat positionnement au sein d'équipes paramédicales. Sur notre université, plusieurs séminaires de formation à l'interprofessionnalité ont été mises en place pour apprendre à communiquer et comprendre l'interprofessionnalité dans sa dimension de rencontrer des cultures organisationnelles et soignantes différentes [24]. Au moyen de jeux de rôles et de simulations, les médecins, infirmières et aides-soignants y apprennent à interagir. Ceci pourrait également aider les jeunes étudiants en médecine (les externes) dans leurs stages [25]. En ce qui concerne les problèmes relationnels entre les internes eux-mêmes et entre les internes et les médecins séniors, plusieurs facteurs semblaient favoriser ces situations et notamment les facteurs 
Vécu au travail des internes

organisationnels au travail. La charge de travail, l'indisponibilité des médecins pour échanger autour des situations ont déjà été décrites. Le nouvel internat ne devrait pas résoudre toutes ces tensions dans la mesure où la charge de travail du médecin sénior ne sera pas réduite ; en d'autres termes, il n'y a pas de réflexion hospitalière à propos de la prise en compte de la formation dans le temps de travail. Certains médecins, dans une étude qualitative, déploraient de ne pas avoir suffisamment de temps pour bien former les internes, expliquant que finalement, avoir un interne dans le service pouvait augmenter la quantité de travail et non la diminuer [26]. Ainsi, le vécu au travail des internes semblerait lié à celui des médecins séniors et, en conséquence, aux conditions de travail en général dans les lieux de stages. La prévention passerait alors par une amélioration de ces conditions de travail, notamment en incluant dans les temps de travail les temps d'échanges et de formations entre médecins, ou en valorisant le temps de formation à l'hôpital.

Conclusion :

Cette étude qualitative apportait un éclairage nouveau sur le vécu des internes au travail, tant à l'hôpital que dans leurs études. Les internes ont exprimé de nombreuses difficultés relationnelles, mais aussi un fort soutien de la part de leurs collègues. La qualité des relations, par ce contraste, recelait un caractère critique pour la qualité de vie au travail des internes, que ce soit des relations entre médecins ou avec les équipes paramédicales. Cette étude permettrait de reprendre les études qualitatives en orientant la méthodologie selon le vécu des internes. Elle constituait d'autre part un état de comparaison pour évaluer les changements qui s'opéreront dans les années à venir pour les internes français, avec la réforme qui se met en place en 2018. 
Vécu au travail des internes

Remerciements: Les auteurs remercient les internes et leurs associations pour leur contribution à la diffusion de l'information de cette étude, ainsi que le doyen de notre faculté, le Pr Berthou, pour son accueil et son écoute.

Bibliographie :

[1] Kerrien M, Pougnet R, Garlantézec R, Pougnet L, Le Galudec M, Loddé B et al. Prévalence des troubles anxieux, de l'humeur et de la souffrance au travail des internes. Presse Med 2015;44(4 Pt 1):e84-91

[2] Sameer-ur-Rehman S, Kumar R, Siddiqui N, Shahid Z, Syed S, Kadir M. Stress, job satisfaction and work hours in medical and surgical residency programmes in private sector teaching hospitals of Karachi, Pakistan. J Pak Med Assoc 2012; 62 (10): 1109-12

[3] Waldman SV, Diez JC, Arazi HC, Linetzky B, Guinjoan S, Grancelli H. Burnout, perceived stress, and depression among cardiology residents in Argentina. Acad Psychiatry 2009; 33 (4): 296-301

[4] Fahrenkopf AM, Sectish TC, Barger LK, Sharek PJ, Lewin D, Chiang VW et al. Rates of medication errors among depressed and burnt out residents: prospective cohort study. BMJ 2008; 336 (7642): 488-91.

[5] Pougnet R, Pougnet L. Troubles anxieux, de l'humeur et syndrome d'épuisement professionnel des internes en médicine: revue de la littérature 2004-2014. J Med Liban $2017 ; 65(1): 35-43$ 
Vécu au travail des internes

[6] Blanchard P, Truchot D, Albiges-Sauvin L, Dewas S, Pointreau Y, Rodrigues M et al. Prevalence and causes of burnout amongst oncology residents: A comprehensive nationwide cross-sectional study. Eur J Cancer 2010; 46 (15): 2708-15.

[7] Cochidon C. Prévalence des troubles de santé mentale et conséquences pour l'activité professionnelle en France dans l'enquête santé mentale en population générale : images et réalité. Santé Travail de l'InVS 2007. Disponible sur: http://opac.invs.sante.fr/doc_num.php?explnum_id=3595

[8] Blanchet A, Gotman A. L'enquête et ses méthodes : l'entretien. Paris : Armand Colin; 2007.

[9] Paillé Pierre, Alex Mucchielli. L'analyse qualitative en sciences hymaines et sociales.Paris : Armand Colin;2005

[10] Hernandez E, Bagourd E, Tremeau AL, Bolot AL, Laporte C, Moréno JP. Perspective de l'acquisition des compétences du métier de médecin généraliste par les internes de médecine générale : enquête nationale. Pédagogie Médicale 2017 ;18(3)109-20

[11] Rich A, Viney R, Needleman S, Griffin A, Woolf K. 'You can't be a person and a doctor': the work-life balance of doctors in training-a qualitative study. BMJ Open 2016;6(12):e013897.

[12] Siegrist J. Adverse health effects of high-effort/low-reward conditions. J Occup Health Psychol 1996; 1(1):27-41.

[13] Li J, Weigl M, Glaser J, Petru R, Siegrist J, Angerer P. Changes in psychosocial work environment and depressive symptoms: a prospective study in junior physicians. Am J Ind Med 2013; 56(12):1414-22 
Vécu au travail des internes

[14] Surman G, Goldacre MJ, Lambert TW. UK-trained junior doctors' intentions to work in UK medicine: questionnaire surveys, three years after graduation. $\mathrm{J} R$ Soc Med 2017;110(12):493-500

[15] Maisonneuve JJ, Lambert TW, Goldacre MJ. Doctors' views about training and future careers expressed one year after graduation by UK-trained doctors: questionnaire surveys undertaken in 2009 and 2010. BMC Med Educ 2014;14:270

[16] Maisonneuve JJ, Lambert TW, Goldacre MJ. Doctors' views about training and future careers expressed one year after graduation by UK-trained doctors: questionnaire surveys undertaken in 2009 and 2010. BMC Med Educ 2014;14:270

[17] Pougnet R, Di Costanzo LP, Kerrien M, Jousset D, Loddé B, Dewitte JD et al. Occupational factors for mood and anxiety disorders among junior medical doctors. Med Lav 2015;106(5):386-93.

[18] Ochsmann E, Lang J, Drexler H, Schmid K. Stress and recovery in junior doctors. Postgrad Med J 2011; 87 (1031): 579-84. 17.

[19] Golub JS, Weiss PS, Ramesh AK, Ossoff RH, Johns MM 3rd. Burnout in residents of otolaryngology-head and neck surgery: a national inquiry into the health of residency training. Acad Med 2007; 82 (6): 596-601.

[20] Hannan E, Breslin N, Doherty E, McGreal M, Moneley D, Offiah G. Burnout and stress amongst interns in Irish hospitals: contributing factors and potential solutions. Ir J Med Sci 2018;187(2):301-307

[21] Buddeberg-Fischer B, Stamm M, Buddeberg C, Klaghofer R. Chronic stress experience in young physicians: impact of person- and workplace-related factors. Int Arch Occup Environ Health 2010; 83 (4): 373-9. 
Vécu au travail des internes

[22] Borrott N, Kinney S, Newall F, Williams A, Cranswick N, Wong I et al. Medication communication between nurses and doctors for paediatric acute care: An ethnographic study. J Clin Nurs 2017;26(13-14):1978-1992

[23] Gunasingam N, Burns K, Edwards J, Dinh M, Walton M. Reducing stress and burnout in junior doctors: the impact of debriefing sessions. Postgrad Med J 2015;91(1074):182-7

[24] Milne J, Greenfield D, Braithwaite J. An ethnographic investigation of junior doctors' capacities to practice interprofessionally in three teaching hospitals. J Interprof Care 2015;29(4):347-53

[25] Le Provost AS, Loddé B, Pietri J, De Parscau L, Pougnet L, Dewitte JD et al. Souffrance au travail des étudiants en medicine: etude qualitative par entretiens semi-dirigés. Rev Med Brux 2018;39(1):6-14

[26] Pougnet R, Pougnet L, Loddé B, Dewitte JD. Travailler à l'hôpital est riche de sens et source de mal être: analyse qualitative auprès de 9 médecins hospitaliers. Presse Med 2016;45(7-8 Pt 1):e233-41 
Figure 1: Carte heuristico-conceptuelle du vécu des internes en médecine au travail. 
Tableau 1: Variation maximale dans l'échantillon.

\begin{tabular}{|c|c|c|c|c|c|c|c|c|c|c|}
\hline Sujets & Sexe & Spécialité & $\begin{array}{c}2^{\mathrm{e}} \\
\text { année }\end{array}$ & $\begin{array}{c}3^{\mathrm{e}} \\
\text { année }\end{array}$ & $\begin{array}{c}4^{\mathrm{e}} \\
\text { année }\end{array}$ & $\begin{array}{l}\text { Changement } \\
\text { de spécialité }\end{array}$ & $\begin{array}{l}\text { Redou- } \\
\text { blement }\end{array}$ & $\begin{array}{l}\text { Stage } \\
\text { CHRU }\end{array}$ & $\begin{array}{l}\text { Autres } \\
\text { stages }\end{array}$ & Enfants \\
\hline 1 & $\mathrm{M}$ & Généraliste & & & $\mathrm{X}$ & & $X$ & $X$ & $X$ & \\
\hline 2 & $\mathrm{~F}$ & Spécialiste & & $\mathrm{X}$ & & $\mathrm{X}$ & & $\mathrm{X}$ & $\mathrm{X}$ & \\
\hline 3 & $\mathrm{~F}$ & Spécialiste & $\mathrm{X}$ & & & & $\mathrm{X}$ & & $\mathrm{X}$ & $\mathrm{X}$ \\
\hline 4 & $\mathrm{~F}$ & Spécialiste & & & $\mathrm{X}$ & & & $\mathrm{X}$ & $\mathrm{X}$ & \\
\hline 5 & $\mathrm{M}$ & Spécialiste & & & $\mathrm{X}$ & & & $\mathrm{X}$ & $\mathrm{X}$ & \\
\hline 6 & $\mathrm{M}$ & Généraliste & & $\mathrm{X}$ & & & & $\mathrm{X}$ & $\mathrm{X}$ & \\
\hline 7 & $F$ & Spécialiste & & & & & & $\mathrm{X}$ & $\mathrm{X}$ & \\
\hline 8 & $\mathrm{~F}$ & Généraliste & & & $\mathrm{X}$ & & $\mathrm{X}$ & $\mathrm{X}$ & $\mathrm{X}$ & $\mathrm{X}$ \\
\hline 9 & $\mathrm{M}$ & Spécialiste & & $X$ & & $X$ & $\mathrm{X}$ & $\mathrm{X}$ & $\mathrm{X}$ & \\
\hline 10 & $\mathrm{~F}$ & Spécialiste & & & $\mathrm{X}$ & & & $X$ & $\mathrm{X}$ & \\
\hline
\end{tabular}

